\title{
Editorial Note: Emotion-Aware Healthcare Systems Integrating with Wearable and Cloud Computing
}

Multimedia Tools and Applications gratefully acknowledges the editorial work of the scholars listed below on the special issue entitled, "Emotion-aware Healthcare Systems Integrating with Wearable and Cloud Computing."

Of 33 papers submitted to this issue, 11 were eventually accepted after a stringent peer-review process.

\section{Yin Zhang (Corresponding Guest Editor)}

Zhongnan University of Economics and Law, China

yin.zhang.cn@iee.org

Giancarlo Fortino

University of Calabria, Italy

giancarlo.fortino@unical.it

Long $\mathrm{Hu}$

The University of British Columbia, Canada

longhu.cs@gmail.com

Kai Lin

University of Pennsylvania, USA

link@dlut.edu.cn

Publisher's Note Springer Nature remains neutral with regard to jurisdictional claims in published maps and institutional affiliations. 